\title{
V. Krystallographische Untersuchung einiger organischen Verbindungen.
}

\author{
Von
}

c. Haushofer in München.

(Mit 7 Holzschnitten.)

\section{Dicyanamid. $\mathrm{C}_{2} \mathrm{~N}_{4} \mathrm{H}_{4}$.}

Dargestellt von Dr. P. 'T a t a rin of im Laboratorium der techn. Hochschule München.

Monosymmetrisch. $a: b: c=1,1150: 1: 0,8055 . \quad \beta=64^{0} 47^{\prime}$.

Beobachtete. Formen: $\infty P(110)=p, \infty P \infty(100)=a, o P(001)$ $=c,-P(111)=n, \infty R 3(130)=q,-\frac{1}{2} P \infty(102)=d$. Die untersuchten Krystalle besitzen zweierlei Ausbildungsweise : die kleineren, in Bezug auf ihre Flächenbeschaffenheit vollkommeneren, sind flachprismatisch nach der Verticalaxe und durch Vorherrschen von $a$ (Fig. 1); die Flächen $a$ vertical gestreift, die Fläche $d$ meist etwas gewölbt und nicht genau zu messen.

Fig. 1.

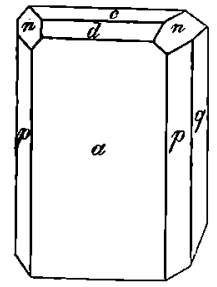

Fig. 2.

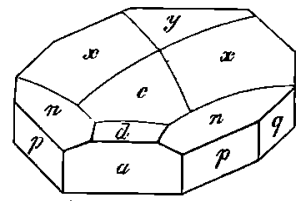

Bei der zweiten Form der Krystalle (Fig. 2) walten die basische Fläche und die ihr benachbarten vor; dieselben sind stark aufgewölbt und verlaufen dergestalt in einander, dass die Krystalle einen linsenförmigen Habitus gewinnen. Nur die Flächen der prismatischen Żone sind eben und scharf begränzt. 


\begin{tabular}{|c|c|c|}
\hline & Beobachtel: & Berechnet \\
\hline$(100)$ & $(001)={ }^{*} 64^{0} 47^{\prime}$ & - \\
\hline 007 & $(110)=* 45^{0} 15^{\prime}$ & - \\
\hline 00.1$)$ & $(111)={ }^{*} 36^{0} 49^{\prime}$ & - \\
\hline 100 & $(102)=47026^{\prime}$ appr. & $48^{0} \quad 8^{\prime}$ \\
\hline$(110)$ & $(001)=72^{0} 33^{\prime}$ & $72^{0} .32^{\prime}$ \\
\hline$(110)$ & $(1 \bar{T} 0)=90^{\circ} 30^{\prime}$ & $90^{\circ} 30^{\prime}$ \\
\hline$(100)$ & $(130)=71^{0} 42^{\prime}$ & $71^{0} 43^{\prime}$ \\
\hline
\end{tabular}

Sehr vollkommen spaltbar nach $a(100)$.

2. Imidopropionnitril. $C_{6} H_{9} N_{3}$.

Dargestellt von Dr. C. Passavant im Laboratorium d. techn. Hochschule München.

Monosymmetrisch. $a: b: c=1,0856: 1: 1,2469 . \beta=70^{0} 21^{\prime}$. Wasserhelle luftbeständige Krystalle der Gombination (Fig. 3) : oP (001)

Fig. 3.

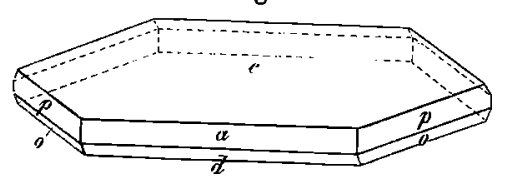

$=c, \infty P \infty(100)=a, \infty P(110)$ $=p, \overline{\boldsymbol{P}} \infty(10 \bar{T})=d, \boldsymbol{P}(11 \bar{T})=0$. 'Dưnn tafelförmig nach $c$, meist nach der Axe $b$ verlängert, seltener nach derselben vollkommen prismatisch. Stark glasglänzend.

Gemessen :

\begin{tabular}{|c|c|c|}
\hline 001$)(100)=$ & $*^{*} 70^{0} 21^{\prime}$ & - \\
\hline$(100)(110)=$ & $* 45^{0} 38$ & - \\
\hline 100) $(10 T)=$ & $49013^{\prime}$ & - \\
\hline 1) $(110)=$ & $76^{\circ} 35$ & $76^{\circ} 24^{\prime}$ \\
\hline 10) $(\overline{1} 10)=$ & - & $8^{0} 44^{\prime}$ \\
\hline 1) $(\bar{T} 11)=$ & $68^{0} 45^{\prime}$ & $68^{0} 59^{\prime}$ \\
\hline
\end{tabular}

Sehr vollkommen spaltbar nach o $P$.
Berechnet:

$89^{2}$

3. Orthonitrozimmtsäureäthylester. $\mathrm{C}_{9} \mathrm{H}_{6}\left(\mathrm{NO}_{2}\right) \mathrm{O}_{2}, \mathrm{C}_{2} \mathrm{H}_{5}$.

Dargesteltt von Dr. L e nk im Laboratorium der technischen Hochschule München.

Rhombisch. $a: b: c=0,5245: 1: 0,8651$.

Blassgelbe durchsichtige Krystalle der Combination (Fig. 4) : o $P$ (001) $=c, \boldsymbol{P}(111)=p, \infty \overline{\boldsymbol{P}} \infty(100)=a, \breve{P} \infty(011)=d ;$ tafelförmig nach der Basis, welche meistens treppenförmig verlieft. Kleinere Krystalle zeigten nur die Combination $P$. o $P$. 


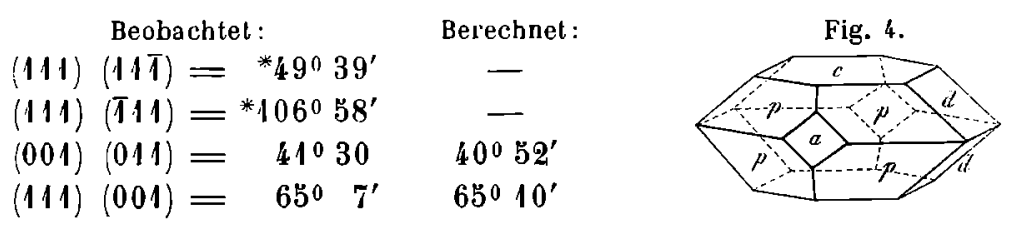

4. Methyluramin-Platinchlorid. $\left(C_{2} \mathrm{H}_{3} \mathrm{~N}_{3} \mathrm{Cl}\right)_{2} \mathrm{Pt} \mathrm{Cl}_{4}$.

Dieses schon früher von Senarmont (Jahresber. Chem. 1857. 542 Anm.) und zuletzt durch v. Kobell (Sitz. Ber. d. k. bair. Akad. d. Wiss. 1870. II, 305) krystallographisch untersuchte Salz wurde neuerdings von Dr. P. Ta tarin of $f$ im Laboratorium der techn. Hochschule zu Munchen in guten Krystallen dargestellt, deren Untersuchung die vorläufigen Angaben von Ko bell's bestätigte.

Krystallsystem monosymmetrisch.

$a: b: c=0,7322: 1: 0,5942 . \quad \beta=76^{0} 47$.

Beobachtete Formen (s. Fig. 5): $\infty P(110)=p$, $\infty R \infty(010)=b, o P(001)=c, P(111)=m,-P(111)$ $=n$.

Die Krystalle sind gewöhnlich tafelförmig nach $b$, meist auch nach der Verticalaxe gestreckt; bisweilen finFig. 5.

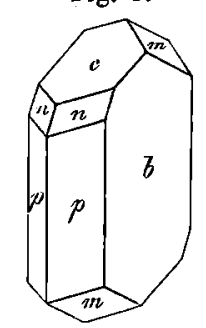
det sich nur die Combination $\infty P$.oP, rhomboederähnlich ausgebildet; am häufigsten ist die Form $\infty P, \infty R \infty, o P$; die Pyramidenflächen fand ich nur an zwei Krystallen.

\begin{tabular}{|c|c|c|}
\hline & messen : & Berech \\
\hline 110) & $(1 \overline{1} 0)={ }^{*} 70^{0} 58^{\prime}$ & - \\
\hline 001$)$ & $(111)={ }^{*} 39^{0} 33^{\prime}$ & - \\
\hline 001) & $(110)=* 79^{0} 16^{\prime}$ & - \\
\hline 111) & $(110)=39^{\circ} 46^{\prime}$ & $39^{0} 43^{\prime}$ \\
\hline T11) & $(001)=50^{0} \quad 8^{\prime}$ & $50^{0} 15^{\prime}$ \\
\hline 111) & $(\overline{11} 1)=$ & $44^{0} \quad 2^{\prime}$ \\
\hline (111) & $(1 T 1)=$ & $44^{0} 12^{\prime}$ \\
\hline I11) & $(040)=62^{0} 14^{\prime}$ & $62^{0} 59^{\prime}$ \\
\hline & $(010)=54033^{\prime}$ & $54^{0} 31^{\prime}$ \\
\hline 11) & $(010)=67^{\circ} 48^{\prime}$ & $67054^{\prime}$ \\
\hline
\end{tabular}

Vollkommen spaltbar nach $\infty R \infty$. Dunkelorangegelb ins byacinthrothe; an der Luft nach längerer Zeit sich trübend. 
5. Methyluramin-Goldchlorid. $\left(\mathrm{C}_{2} \mathrm{H}_{8} \mathrm{~N}_{3} \mathrm{Cl}\right) \mathrm{AuCl} \mathrm{Cl}_{3}$.

Dargestellt von Dr. P. Ta tar in off im Laboratorium der techn. Hochschule München.

Rhombisch. $\quad a: b: c=0,9867: 1: 0,4993$.

Die Zeichen der beobachteten Formen, bezogen auf die gewöhniliche Stellung rhombischer Krystalle, sind: $\infty P(110)=p, \infty \bar{P} 2(210)=n$, $\infty \bar{P} \infty(100)=a, \infty \breve{P} \infty(010)=b, \breve{P} \infty(011)=r, \frac{5}{4} \bar{P} \infty(504)=q$.

Die Krystalle (s. Fig. 6) erscheinen gewöhnlich säulenförmig durch Vorwalten zweier Parallelflächen von $\infty P$; die ungleichmässige Ausdehnung

Fig. 6.

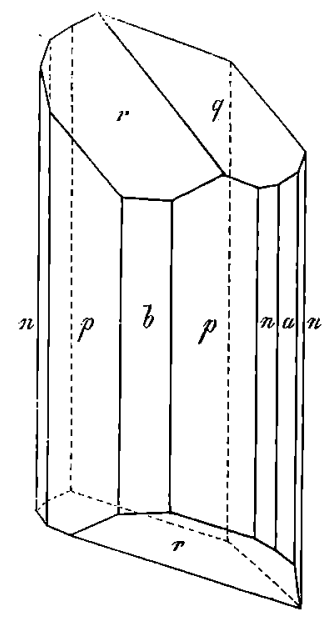
einzelner Flächen der beiden Domen $r$ und $q$ verleiht ibnen einen klinoaxen Habitus; an 8 ringsum ausgebildeten Exemplaren wurden in gleicher Weise n u r die beiden vorderen Flächen von $\breve{\boldsymbol{P}} \infty$ $(0 \bar{T} /$ und $0 \bar{T} \bar{T})$, die rechts oben und links unten liegende Fläche von $\frac{5}{4} \bar{P} \infty$ (also 504 und $\overline{5} 0 \overline{4}$ ) gefunden, wenn man, wie diess in der Zeichnung der grösseren Verständlichkeit wegen geschehen ist, die Krystalle so stellt, dass die Brachydiagonale in die Zeichnungsebene fällt. Nur an zwei Krystallen zeigten sich Andeulungen der ubrigen domatischen Flächen. Auch die Prismenflächen und verticalen Flächenpaare treten sehr ungleichwerthig auf; gewöhnlich dominiren die beiden Gegenflächen ( $(\bar{T} 0)$ und (110). Die Flächen $r$ und $q$ sind meistens schlecht entwickelt; das gilt ganz besonders für $q$, dessen Deutung als $\frac{5}{4} \bar{P} \infty$ nicht ganz zweifellos ist, obwohl die Berechnung aus dem Winkel $a: q=(100)(504)$ zu demselben Werthe führte, wie die aus dem Winkel $q: r=(504):(0 \bar{T} 1)$.

Gemessen :

(010) $(110)=* 45^{0} 23^{\prime}$

(010) $(011)={ }^{*} 63^{0} 28^{\prime}$

(201) $(100)=26^{\circ} 30^{\prime}$

(110) $(210)=190 \quad 0$

(100) $(504)=58023$

(504) $(110)=67056$

(2T0) $(210) \doteq 52013$

(110) $(100)=44^{027}$

(1T0) $(110)=-$

$(110)(\overline{1} 10)=89019$
Berechnet :

$26^{\circ} 16^{\prime}$

$19^{0} 21$

$57045^{\prime}$

67041

52031

$44^{0} 37$

90046

89014

Spaltbarkeit unvollkommen parallel o $P$. - Starker Glasglanz auf 
allen Flächen; dunkelorangegelb, an der Luft unter Zersetzung langsam sich bräunend.

Die Auslöschungsrichtungen liegen auf allen Flächen der prismatischen Zone parallel und senkrecht zu den Prismenkanten; senkrecht zur Prismenaxe geschnittene Lamellen zeigen in Oel beide Axenbilder; die Axenebene liegt parallel $b$.

6. Schleimsaures Natron. $\mathrm{C}_{6} \mathrm{H}_{8} \mathrm{O}_{8} \mathrm{Na}_{2}+5 \mathrm{H}_{2} \mathrm{O}$.

Dargestellt von Hrn. Fr. W. Re i man im Laborat. der lechn. Hochschule München.

Asymmetrisch. $\quad a: b: c=0,6008: 1: 1,3786$.

$$
\begin{aligned}
& \alpha=120^{\circ} 21^{\prime} . \\
& \beta=90^{\circ} 31^{\prime} . \\
& \gamma=110^{\circ} 37^{\prime} .
\end{aligned}
$$

Farblose, ziemlich schnell verwitternde Krystalle von kurzsäulenförmigem Bau (Fig. 7); beobachtete Formen : oP (001) $=c, \infty \bar{P} \infty(100)=a$, $\infty \breve{P} \infty(100)=b, \infty P_{r}^{\prime}(110)=p, ' \breve{P}, \infty(01 \bar{T})=d$. Die Fläche $\infty \mathrm{P}_{\prime}^{\prime}(p)$ meist sehr schmal und nur an zwei Krystallen beobachtet. Das wahrscheinlich in der Zone $a b$ liegende Flächenpaar $x$ war an allen untersuchten Exemplaren entweder drusig rauh oder, wenn glatt, so sehr gewölbt, dass von einer Messung Abstand genommen werden musste.

$$
\begin{aligned}
& (100)(010)={ }^{*} 65^{0} 35^{\prime} \\
& (100)(001)={ }^{*} 76^{0} 37^{\prime} \\
& (010)(001)={ }^{*} 57^{0} 6 \\
& (110)(010)={ }^{*} 39^{0} 23^{\prime} \\
& (001)(011)={ }^{*} 71024 \\
& (100)(01 \overline{1})=51^{\circ} 26^{\prime}, \text { berechnet } 51^{0} 30^{\prime} \\
& (001)(110)=-64^{0} 52
\end{aligned}
$$

Spaltbarkeit nicht zu beobachten. Durch $x$ ein Axenbild sichtbar; der dunkle Balken desselben

Fig. 7.

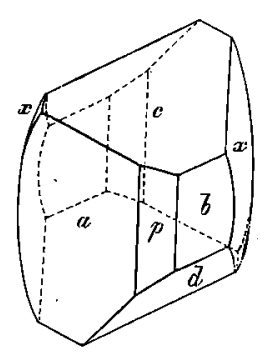
schneidet die Kante $x c$ unter $c .60^{\circ}$. Auslöschungsrichtung auf $a$ fast normal zur Kante $a c$, auf $c$ die Kanle $a c$ unter c $.20^{\circ}$ schneidend. 